\title{
Reducing occupational distress in veterinary medicine personnel with acceptance and commitment training: a pilot
} study

\author{
MB Spitznagela§, ASG Updegraffa ${ }^{\text {a }}$ MP Twohig ${ }^{b}$, MD Carlson $^{c}$ and CM Fulkerson ${ }^{d}$ \\ ${ }^{a}$ Department of Psychological Sciences, Kent State University, Kent, OH, USA \\ ${ }^{b}$ Department of Psychology, Utah State University, Logan, UT, USA \\ c Stow Kent Animal Hospital, Kent, OH, USA \\ d Department of Veterinary Clinical Sciences, College of Veterinary Medicine and Center for \\ Cancer Research, Purdue University, West Lafayette, IN, USA \\ $\S$ Author for correspondence. Email: mspitzna@kent.edu
}

\section{Abstract}

Aims: To determine whether an educational programme targeting the reaction of veterinary medicine personnel to difficult client interactions reduces burden transfer, stress and burnout in veterinary medicine staff.

Methods: Employees of three small animal veterinary hospitals in the south-western United States of America were recruited and randomised to intervention (educational programme; $n=16$ ) or control (no intervention; $n=18$ ) groups. Participants of this randomised, parallel arms trial completed pre-programme assessment including the Burden Transfer Inventory (BTI), Perceived Stress Scale, and Copenhagen Burnout Inventory. Assessment was followed by two, group-format educational sessions, based on acceptance and commitment training, tailored to reducing reactivity to difficult veterinary client interactions (intervention group only). After training was completed, both groups were assessed using the same measures and the intervention participants provided use and acceptability ratings.

Results: Intervention participants rated the programme as useful and appropriate, and reported that programme techniques were used a median of $43(\min 9$, max 68$)$ times during the 2 weeks prior to retesting. Relative to pre-programme scores, median post-programme scores for reaction (subscore of $\mathrm{BTI}$ ) to difficult client interactions decreased in the intervention group (33 vs. 54; $p=0.047$ ), but not in the control group (51 vs. $59 ; p=0.210$ ). Changes in median scores for stress and burnout from pre- to post-programme were non-significant for both groups.

Conclusions: This pilot and feasibility trial showed high rates of acceptability and use by participants, as well as promising reductions in burden transfer. A larger scale clinical trial with follow-up at extended time points is needed to more fully examine the efficacy of this novel programme. 
Clinical relevance: Preliminary findings suggest this programme may be a useful approach to reducing occupational distress for individuals working in the field of veterinary medicine.

Keywords: Acceptance and commitment training, burden transfer, veterinarian stress, veterinarian burnout, veterinarian wellbeing, occupational distress

Abbreviations: ACT: Acceptance and commitment training; CBI: Copenhagen Burnout Inventory; DANCE: Daily hassles, affect, non-adherent/inconsiderate, confrontations, excess communications; PSS: Perceived Stress Scale

\section{Introduction}

Although $80 \%$ of veterinarians describe their occupation as stressful (Bartram and Baldwin 2008; Nett et al. 2015), there remains a dearth of research examining strategies to address this problem (Moir and Van den Brink 2020). Many occupational stressors in veterinary medicine have been identified, including compassion fatigue, moral distress, overwork, fear of complaints, and frustration with unrealistic client expectations and non-compliance (Moses et al. 2018; Van de Grieke et al. 2018; Moir and Van den Brink 2020). At the core of these stressors may often be the difficult interactions that veterinary personnel have with clients who are experiencing caregiver burden (Spitznagel et al. 2017; Spitznagel et al. 2019a), the multi-faceted strain associated with caring for a loved one with an illness (Zarit et al. 1980). Recently, the caregiver burden experienced by veterinary clients with a sick companion animal has been linked to distress in veterinary personnel, a phenomenon termed "burden transfer" (Spitznagel et al. 2019b). This theory of burden transfer posits that caregiver burden in veterinary clients underlies stressful encounters with providers, effectively transferring client burden to veterinary personnel.

We have previously identified specific domains of difficult veterinarian-client interactions through factor analysis, and refer to these in combination as the "burden transfer DANCE": daily hassles (e.g., clients wanting impossible predictions, having difficulty making decisions, following others' advice about patient health needs, shopping around to compare costs), affect (e.g., clients requiring euthanasia counselling, demonstrating anxiety, sadness, grief), nonadherent/inconsiderate behaviours (e.g., clients declining recommended work-up or treatment, noshowing for appointments), confrontations (e.g., clients becoming upset, blaming, refusing to pay for services, making a complaint), and excess communications (e.g., frequent phone or email contact). These behaviours are related to caregiver burden in the veterinary client and significantly predict stress and burnout for veterinarians (Spitznagel et al. 2019b).

Importantly, while the frequency of DANCE interactions correlates with stress and burnout for veterinarians, in most cases, the reaction to these behaviours, or how bothered they feel by them, is a far more critical predictor than the frequency of these events (Spitznagel et al. 2019b). DANCE 
reactivity thus appears to drive the transfer of burden, resulting in negative outcomes. For example, a client under financial stress might refuse a recommended treatment or request euthanasia for a treatable problem (an interaction in the "non-adherence" DANCE category). In this case, the client's burden could transfer to the veterinarian, resulting in moral distress due to the ethical conflict of not being permitted to help an otherwise treatable animal. Or, a client with caregiver burden involving uncertainty about the future may cry while discussing their terminally ill pet (an interaction in the "affect" DANCE category). Through repeated interactions of this nature, client burden might transfer to the veterinarian, resulting in compassion fatigue. DANCE interactions could thus serve as a mechanism for commonly discussed outcomes in veterinary medicine like moral distress (Moses et al. 2018) and compassion fatigue (Cohen 2007). Moreover, the veterinary provider's reaction to other DANCE interactions (e.g., daily hassles, confrontations, excess communication) could extend beyond these outcomes, providing greater insight into mechanisms of occupational distress in the field.

Whereas changing the behaviour of veterinary clients (and thus the frequency of such interactions) might prove difficult, the veterinary medicine worker's reaction to (or how affected they are by) such challenging encounters is a potentially modifiable risk factor for occupational distress. A skillsbased education programme that reduces burden transfer could help protect against negative downstream consequences like stress and burnout. An acceptance and commitment training (ACT) programme targeting reactivity to veterinary client DANCE interactions may be of use to this end. ACT is an evidence-based framework that addresses an individual's relationship with stressful thoughts, feelings, and urges, encouraging emotional acceptance, and a re-focus on taking committed action aligned with values (Hayes 2004). This enables the individual to respond in ways that help them to develop meaning and gain fulfilment in their work instead of simply reacting in the moment. ACT has previously shown effectiveness in workplace contexts (e.g., Hayes et al. 2004; Brinkborg et al. 2011; Pulschen and Pulschen 2015) but has not been used to address burden transfer and has never been applied in veterinary medicine.

The aim of this work was to conduct pilot study of whether an ACT-based educational programme created to specifically target reactivity to DANCE interactions in veterinary medicine personnel working in a small animal setting would reduce burden transfer, stress and burnout. We hypothesised that participants would find the programme appropriate and useful, and that burden transfer, stress, and burnout would be decreased when measured after receiving the educational programme in the intervention group, but not in the control group.

\section{Materials and methods}

\section{Participants}

Participants were employees (veterinarians and support staff) of a small animal veterinary hospital group providing primary care, referral, and emergency care at three locations in the southwestern 
United States of America. Inclusion criteria for the study were: over age 18 and able to speak and comprehend English. Exclusion criteria were working in a position with less than $10 \%$ of activities involving client interaction and not attending work during the study period. Participants were neither screened nor excluded for psychological issues such as depression or anxiety, as such exclusions could limit generalisability to the workforce.

\section{Study design}

This randomised, parallel arms (intervention, control) pilot trial was approved by the Institutional Review Board of Kent State University (Kent, Ohio, USA). The study took place from January to May 2020. To construct study groups in the most generalisable manner, the programme was provided as an educational opportunity for all hospital employees. Those choosing to participate in the programme were given the additional option to provide anonymised data for research. Data were collected through the online data collection platform, Qualtrics (Provo, UT, USA), beginning with informed consent, which noted the study purpose, participant rights, Institutional Review Board approval status, and contact information.

Individuals choosing to be involved in research were allocated to one of two groups via Qualtrics auto-randomisation: the intervention group which received an educational programme based on ACT and the control group which did not. Both groups completed three measures (Burden Transfer Inventory(BTI), Perceived Stress Scale (PSS) and Copenhagen Burnout Inventory (CBI): see below for more details) and provided demographic information in a baseline session (preprogramme). Within 1 month of the baseline session, the intervention group then completed two ACT sessions (see below). Within 1 month of completing the ACT session, both groups completed the measures again in a review session (post-programme). Therefore, the control group completed pre-programme and post-programme measures in the same timeframe as the intervention group, without having completed the ACT programme, which was made available to them following data collection; these individuals were sent a link via email to complete the post-programme measures. Due to the nature of shift work and multiple locations, group sessions could be accessed in-person (baseline session and ACT session 1 only due to the COVID-19 pandemic), live-streaming, or as a recording at a later time. Sessions were delivered by a member of the research team (MBS) and session recordings were checked for fidelity to established protocol by a separate member of the team (ASGU). Participants were eligible for a raffle to win one of three gift cards for an online retailer.

\section{ACT educational programme}

The programme was based on an ACT framework, which combines mindfulness and emotional acceptance education with experiential exercises and techniques for behaviour change (Hayes, 2004). It relies on six inter-related components (Hayes et al. 1999; Bach and Moran 2008; Twohig and Hayes 2008): (1) "being present" which means consciously experiencing internal and external 
events in the moment; (2) "acceptance" which is actively embracing inner events (e.g., thoughts, feelings, or urges) while they are occurring; (3) "defusion" which involves viewing inner events as no more than words, sensations, and images; (4) understanding the "self-as context" which involves observation of inner struggles from an outside perspective; (5) "values" which are areas of importance that an individual identifies and embraces as a guide to action and 6) "committed action" which means behaving in a manner that serves a chosen value.

The protocol utilised in the current work was based on these six core processes, with a focus on identifying DANCE interactions and reducing reactivity during these situations. The interested reader is referred to the Supplementary Information ${ }^{1}$ for more details on session contents. Briefly, participants were taught to not become activated by difficult thoughts and feelings that are part of their daily work with clients, but to let personal values guide them.

Four total group sessions were delivered, spread 1 month apart, with homework assigned to practice skills taught between sessions. The baseline session included an introduction to the study, followed by collection of pre-programme data and then brief education regarding burden transfer. Those assigned to the intervention condition then completed ACT sessions 1 and 2, the "active ingredient" sessions. ACT session 1 focused on skills for being mindfully present, identifying and accepting difficult thoughts, feelings, and urges in the context of DANCE interactions, and separating the self from these inner struggles to understand them as an observer. ACT session 2 helped participants clarify personal values and discussed skills for taking committed action to focus on alternate values as needed during DANCE interactions. In the review session, post-programme measures were completed, followed by a brief review of all material.

\section{Data collected}

Participants in both groups completed the following measures in the baseline (pre-programme) and review (post-programme) sessions. Please see Supplementary Information ${ }^{1}$ for more detail on these measures.

\section{The Burden Transfer Inventory}

The Burden Transfer Inventory (Spitznagel et al. 2019b) measures burden transfer by asking the respondent about the five domains of client DANCE interactions. Higher BTI subscale scores indicate greater frequency of (BTI frequency) or reaction to (BTI reaction) these experiences. This

33-item measure demonstrates adequate to excellent internal consistency across subscales with Cronbach's $\alpha$ ranging from 0.58 to 0.88 across subscales for frequency and reaction, and from 0.92 to 0.94 for combined subscales.

The Perceived Stress Scale 
The Perceived Stress Scale (Cohen et al. 1983) is a widely-used measure of stress perception, addressing degree of current stress and feelings that life is unpredictable or overloaded on a 10question scale. Higher PSS scores indicate greater current stress. This measure demonstrates internal consistency $(\alpha=0.68-0.78)$ and convergent validity with anxiety and anger $(\beta=0.54-0.68)$ (Perera et al. 2017).

\section{The Copenhagen Burnout Inventory}

The Copenhagen Burnout Inventory (Kristensen et al. 2005) is a 19-item scale addressing personal, work, and client-related burnout, with higher CBI scores indicating greater current burnout. Psychometric properties of the $\mathrm{CBI}$ include high internal consistency ( $\alpha=0.85-0.87)$ and construct validity including negative correlations with measures of mental health and vitality $(r=0.39-0.75)$. Given the focus of the current study, personal burnout was omitted; work- and client-related burnout subscales were averaged for a single variable.

Participants also provided additional data through self-reported demographic information, specifically gender (multiple choice), age (continuously numbered slide bar), race or ethnicity (multiple choice), nature of employment (multiple choice), and percent of job activities involving client interaction (continuously numbered slide bar). Due to the COVID-19 pandemic and hospitalwide shift to curb-side service (i.e., the animal is brought into the hospital while the owner waits in the car and speaks to staff primarily by phone) during the study period, percent of job activities involving client interaction was asked at both timepoints.

At the post-programme measurement, those who participated in the programme were also asked to report appropriateness and usefulness of the material presented (scale of 1-5 where $1=$ poor and $5=e x c e l l e n t)$, as well as how frequently during the preceding 2 weeks they used ACT techniques taught in the programme.

\section{Statistical analysis}

Demographic information and primary variables were characterised using descriptive statistics (percentage for categorical data, median (min, max) for continuous data). Due to small sample sizes in each cell, non-parametric tests and individual group comparisons were utilised. The paired samples Wilcoxon Signed Rank test was used to test if outcome variables (BTI reaction, BTI frequency, PSS, CBI) decreased from pre-programme to post-programme in each group. Due to the shift to curb-side service during the study period, both BTI frequency and reaction were examined. Given that no prior work has examined effects of this educational programme, interpretation of results relied on two-tailed alpha $(P<0.05)$, as well as percent change, effect size, and directionality. All analyses were conducted using SPSS 26.0 (Armonk, NY, USA).

\section{Results}




\section{Participants}

Measures were completed at both timepoints (pre- and post-programme) by 38 individuals; three were removed from analyses due to reporting $<10 \%$ of job activities involving client interaction, and one was removed due to being away from work during the 2-week period prior to post-test. The final analytic sample was 34 (intervention $n=16$; control $n=18$ ). Both groups were comprised primarily of females identifying as white, with a mean age of approximately 35 years (Table 1). Positions of employment included veterinarians, technicians, assistants, customer service representatives, and management. Aligning with the shift to curb-side service during this period, the median percentage of time participants reported spending in client interactions was lower in the post-programme data than pre-programme data for the control group (56 ( $\min 20$, max 100)\% vs. $74(\min 31, \max 100) \% ; p=0.012)$. For the intervention group, there was a trend towards a lower percentage post-programme $(70(\min 21, \max 100) \%$ vs. $80(\min 27, \max 100) \% ; p=0.091)$. No variables differed significantly between the two groups.

\section{Acceptability of ACT educational programme}

At post-programme session, $100 \%$ of the participants randomised to the intervention group reported that the level and content of the material presented was appropriate, and $93.3 \%$ indicated the material was useful (i.e., ratings of "Average," "Above Average," or "Excellent" for each category; Table 2). Participants reported using techniques taught during the programme a median of 43 ( $\min 9, \max 68)$ times during the preceding 2 weeks.

\section{Effect of programme on burden transfer, stress, and burnout}

Median BTI frequency scores were lower when measured post-programme compared to preprogramme for both the intervention group (64 vs. 68; $p=0.041$ ) and the control group (58 vs. 62; $\mathrm{p}=0.006$ ) (Table 3 ). In contrast, median BTI reaction scores were only lower post-programme compared to pre-programme for the intervention group (33 vs. 54; $\mathrm{p}=.047$ ). Changes in median scores for stress (PSS) and burnout (CBI) from pre- to post-programme did not reach statistical significance (see Table 3).

\section{Discussion}

The present study was a feasibility and pilot trial of a new ACT-based educational programme aimed at decreasing burden transfer, stress, and burnout in veterinary medicine personnel via reduction of reactivity to DANCE interactions with clients, which prior research shows are associated with stress and burnout in veterinarians (Spitznagel et al. 2019b). Overall, participants found the programme acceptable and used the skills that were taught. While frequency of DANCE interactions decreased in both the intervention and control groups with the change to curb-side service and lower general client interaction, reaction to DANCE interactions was significantly 
decreased in the intervention group only. Measures of stress and burnout did not show statistically significant differences.

As a pilot trial, it was important to establish the acceptability of this programme for its intended audience of veterinary personnel. Both appropriateness and usefulness of the programme were positively endorsed by a majority of participants. Use of techniques learned in the programme was high at post-programme testing, reflecting use of these skills approximately three times per day on average. Findings suggest the programme content was well-received. It will be important to continue examining acceptability in future samples, but the likelihood of generalisability is considered good, given that the recruitment site spanned a range of services (primary, referral, and emergency care), and the sample represented a range of positions (veterinarians, technicians, assistants, customer service representatives, and management).

As predicted, the programme reduced reaction to DANCE interactions (BTI reaction). This did not occur in the control group. We note that, while it was not statistically significant, a lower median raw score was also observed for the control group; this might be explained by the decreased frequency of DANCE interactions observed in both groups. Although not an original hypothesis, the decreased frequency of DANCE interactions aligned with overall decreased client interactions due to moving to curb-side service during this period. It is possible that all participants experienced slightly greater capacity to cope with difficult client interactions due simply to having fewer of them. However, those who completed the programme showed a large, statistically significant change in BTI reaction, while the control group did not. It thus appears that the programme was successful in reducing burden transfer from these difficult situations.

We did not find evidence to support the prediction that reduction of stress and burnout would be observed in the intervention group. Burden transfer has previously been posited as a mechanism for stress and burnout (Spitznagel et al. 2019b), thus it would make sense if consistently reduced reactivity to DANCE interactions is needed to cumulatively contribute to detectable reductions in stress and burnout over time. Extended follow-up to examine this question is needed. However as the direction of change in median raw scores for stress and burnout was consistent with our hypotheses, future work should examine whether this programme might lead to significantly decreased stress and burnout at later timepoints.

Limitations of the current work are present. The COVID-19 pandemic impacted findings in ways that could be measured (e.g., decreased client interaction), but also potentially in ways not captured here (e.g., personal life changes). Data are thus interpreted with caution, noting that future work is needed to examine these issues under less unusual circumstances. Because the pandemic led to an inability to gather for in-person group sessions midway through the programme, it is also not possible to determine if any differences would be present among those completing the programme in-person, live-streaming, or via recording at a later time. This is a 
future direction that holds important implications for delivery of such a service. The sample also lacks diversity, which is not different from the population of veterinary medicine personnel in the USA (Elmore 2020) where the study was conducted, but may not reflect experiences of individuals who are under-represented in the field. Finally, the small sample size and lack of extended followup are acknowledged. A randomised, controlled trial is needed, optimally with a larger sample, extended monitoring period, and more sophisticated analytic procedures such as mixed linear modelling and path analysis capable of detecting small changes and moderators of benefit from this programme. Regardless of limitations, given that evidence for programmes reducing occupational distress in veterinary medicine by other methods is severely lacking (Moir and Ven den Brink 2020), this work represents an exciting new direction.

In conclusion, the ACT-based educational programme to reduce reactivity during difficult client interactions developed for the current study represents a novel approach to decreasing occupational distress in the field of veterinary medicine. This pilot and feasibility trial showed high rates of acceptability and use by participants, as well as promising reductions in burden transfer, despite confounds introduced by COVID-19. Future work should include a larger scale, controlled trial to examine if this education programme can successfully reduce burden transfer, and in turn, stress and burnout, in veterinary medicine personnel.

\section{Acknowledgements}

The authors would like to acknowledge Angela Thorp, AAS, CVT, VTS (ECC, SAIM) for her assistance with data collection.

This work was funded through a grant to the first author (MBS) from the Kent State University Research Council.

\section{References}

*Bach P, Moran DJ. ACT in Practice: Case Conceptualization in Acceptance and Commitment Therapy. New Harbinger Press, Reno, NV, USA, 2008

Bartram DJ, Baldwin DS. Veterinary surgeons and suicide: influences, opportunities, and research directions. Veterinary Record 162, 36-40, 2008.

Bartram DJ, Baldwin DS. Veterinary surgeons and suicide: a structured review of possible influences on increased risk. Veterinary Record 166, 388-97, 2010.

\section{Brannick EM, DeWilde CA, Frey E, Gluckman TL, Keen JL, Larsen MR, Mont SL, Rosenbaum} MD, Stafford JR, Helke KL. Taking stock and making strides toward wellness in the veterinary workplace. Journal of the American Veterinary Medical Association 247, 739-42, 2015. 
Brinkborg H, Michanek J, Hesser H, Berglund G. Acceptance and commitment therapy for the treatment of stress among social personnel: a randomized controlled trial. Behaviour Research and Therapy 49, 389-98, 2011

Cohen SP. Compassion fatigue and the veterinary health team. Veterinary Clinics of North America: Small Animal Practice 37, 123-34, 2007

Cohen S, Kamarck T, Mermelstein R. A global measure of perceived stress. Journal of Health and Social Behavior 24, 386-96, 1983

Elmore RG. The lack of racial diversity in veterinary medicine. Journal of the American Veterinary Medical Association 222, 24-6, 2020

*Hayes SC. Acceptance and Commitment Therapy and the New Behavior Therapies: Mindfulness, Acceptance and Relationship. Guilford Press, New York, NY, USA, 2004.

Hayes SC, Bissett R, Roget N, Padilla M, Kohlenberg BS, Fisher G, Masuda A, Pistorello J, Rye AK, Berry K, et al. The impact of acceptance and commitment training and multicultural training on the stigmatizing attitudes and professional burnout of substance abuse counselors. Behavior Therapy 35, 821-35, 2004

*Hayes SC, Strosahl K, Wilson KG. Acceptance and Commitment Therapy: An Experiential Approach to Behavior Change. Guilford Press, New York, NY, USA, 1999

Kristensen TS, Borritz M, Villadsen E, Christensen KB. The Copenhagen Burnout Inventory: a new tool for the assessment of burnout. Work Stress 19, 192-207, 2005.

Moir FM, Van den Brink A. Current insights in veterinarians' psychological well-being. New Zealand Veterinary Journal 68, 3-12, 2020

Moses L, Malowney MJ, Boyd JW. Ethical conflict and moral distress in veterinary practice: a survey of North American veterinarians. Journal of Veterinary Internal Medicine 32, 2115-22, 2018

Nett RJ, Witte TR, Holzbauer SM, Elchos BL, Campagnolo ER, Musgrave KJ, Carter KK, Kurkijian KM, Vanicek CF, O'Leary DR et al. Risk factors for suicide, attitudes toward mental illness, and practice-related stressors among US veterinarians. Journal of the American Veterinary Medical Association 247, 945-55, 2015

\section{Perera MJ, Brintz CE, Birnbaum-Weitzman O, Penedo FJ, Gallo LC, Gonzalez P, Gouskova} N, Isasi CR, Navas-Nacher EL, Perreira KM, et al. Factor structure of the Perceived Stress Scale-10 (PSS) across English and Spanish language responders in the HCHS/SOL Sociocultural Ancillary Study. Psychological Assessment 29, 320-8, 2017 
Pulschen S, Pulschen D. Preparation for teacher collaboration in inclusive classrooms-stress reduction for special education students via acceptance and commitment training: a controlled study. Journal of Molecular Psychiatry 3, 8, 2015

Spitznagel MB, Jacobson DM, Cox MD, Carlson MD. Caregiver burden in owners of a sick companion animal: a cross-sectional observational study. Veterinary Record 181, 321, 2017

Spitznagel MB, Cox MD, Jacobson DM, Albers A, Carlson MD. Assessment of caregiver burden and associations with psychosocial function, veterinary service use, and factors related to treatment plan adherence among owners of dogs and cats. Journal of the American Veterinary Medical Association 254, 124-32, 2019a

Spitznagel MB, Ben-Porath YS, Rishniw M, Kogan LR, Carlson MD. Development and validation of a Burden Transfer Inventory measure for predicting veterinarian stress related to client behavior. Journal of the American Veterinary Medical Association 254, 133-44, 2019b

*Twohig M, Hayes SC. ACT Verbatim for Depression and Anxiety: Annotated Transcripts for Learning Acceptance and Commitment Therapy. Context Press, Oakland, CA, USA, 2008

Van de Grieke OH, Clark MA, Witte TK, Nett RJ, Moeller AN, Stabler ME. Development of a taxonomy of practice-related stressors experienced by veterinarians in the United States. Journal of the American Veterinary Medical Association 252, 227-33, 2018

Zarit SH, Reever KE, Bach-Peterson J. Relatives of the impaired elderly: correlates of feelings of burden. The Gerontologist, 20, 649-55, 1980

* Non-peer-reviewed

Submitted 7 September 2020

Accepted for publication 26 May 2020 
Table 1. Demographic information reported by veterinary staff of three small animal hospitals in the southwestern United States who had (intervention group; $n=16$ ) or had not (control group; $n=18$ ) completed an educational programme based on acceptance and commitment training targeting difficult client interactions in veterinary medicine.

\begin{tabular}{lccc}
\hline Variable & Intervention & Control & Total (n=34) \\
\hline Age (years) & $36(23,54)$ & $36(21,58)$ & $35(21,58)$ \\
Race/ethnicity & & & \\
Caucasian/white & $12(75 \%)$ & $16(88.9 \%)$ & $28(84.2 \%)$ \\
Latinx/Hispanic & $2(12.5 \%)$ & 0 & $2(5.9 \%)$ \\
Native American & $2(12.5 \%)$ & 0 & $2(5.9 \%)$ \\
Asian American & 0 & 0 & 0 \\
African American/Black & 0 & 0 & 0 \\
other & 0 & $2(11.1 \%)$ & $2(5.9 \%)$ \\
Gender & 0 & $2(11.1 \%)$ & 0 \\
Male & $16(100 \%)$ & $16(88.9 \%)$ & $32(94.1 \%)$ \\
Female & 0 & 0 & 0 \\
Prefer not to say & $7(1,33)$ & $8(1,27)$ & $8(1-, 3)$ \\
Years in field & & & $8(23.5 \%)$ \\
Position & $4(25.0 \%)$ & $4(22.2 \%)$ & $13(38.2 \%)$ \\
Veterinarian & $5(31 / 1 \%)$ & $8(44.4 \%)$ & $2(5.9 \%)$ \\
Technician & 0 & $2(11.1 \%)$ & $6(17.6)$ \\
Assistant & $4(25.0 \%)$ & $2(11.1 \%)$ & $2(5.9 \%)$ \\
Customer service & $2(12.5)$ & 0 & $2(5.9 \%)$ \\
Management & $1(6.3 \%)$ & $1(5.6 \%)$ & \\
Other & $00 \%)$ & & \\
\hline
\end{tabular}

a Median (min, max) is presented for continuous variables and number (\%) is reported for categorical variables. 
Table 2. Number (\%) of veterinary staff (intervention group; $n=16$ ) of three small animal hospitals in the southwestern United States who completed an educational programme based on acceptance and commitment training who reported their assessment of the acceptability of the programme.

\begin{tabular}{lcccccc}
\hline Acceptability rating & Poor & Fair & Average & Above average & Excellent & $\begin{array}{c}\text { Omitted } \\
\text { item }\end{array}$ \\
\hline Appropriateness & 0 & 0 & $2(12.5 \%)$ & $6(37.5 \%)$ & $7(41.8 \%)$ & $1(6.3 \%)$ \\
Usefulness & 0 & $1(6.3 \%)$ & $2(12.5 \%)$ & $5(31.3 \%)$ & $7(41.8 \%)$ & $1(6.3 \%)$ \\
\hline
\end{tabular}


Table 3. Median (min, max) scores for the Burden Transfer Inventory, Perceived Stress Scale and Copenhagen Burnout Inventory measures which were administered to the veterinary staff of three small animal hospitals in the southwestern United States who had (intervention group; $n=16$ ) or had not (control group; $n=18$ ) completed an educational programme based on acceptance and commitment training before (pre-programme) and after (post-programme) completing the programme.

\begin{tabular}{|c|c|c|c|c|c|c|}
\hline Measures & Pre-programme & Post-programme & $\begin{array}{l}\text { Percent } \\
\text { change }^{\mathrm{a}}\end{array}$ & Z-score & $\mathbf{r}$ & $\begin{array}{c}\mathrm{P}- \\
\text { value }\end{array}$ \\
\hline \multicolumn{7}{|l|}{ Perceived Stress Scale } \\
\hline Control group & $21(8,30)$ & $21(12,27)$ & 0 & -0.24 & 0.04 & 0.813 \\
\hline Intervention group & $19(9,26)$ & $17(9,27)$ & $-11 \%$ & -0.85 & 0.16 & 0.395 \\
\hline \multicolumn{7}{|l|}{$\begin{array}{l}\text { Copenhagen Burnout } \\
\text { Inventory }\end{array}$} \\
\hline Control group & $46(17,93)$ & $50(13,87)$ & & & & 0.777 \\
\hline Intervention group & $54(16,92)$ & $48(13,89)$ & $-11 \%$ & -0.35 & 0.06 & 0.730 \\
\hline \multicolumn{7}{|c|}{ Burden Transfer Inventory } \\
\hline \multicolumn{7}{|l|}{ Frequency } \\
\hline Control group & $62(0,104)$ & $58(0,96)$ & $-6 \%$ & -2.86 & 0.45 & 0.006 \\
\hline Intervention group & $68(33,99)$ & $64(29,95)$ & $-6 \%$ & -2.04 & 0.37 & 0.041 \\
\hline \multicolumn{7}{|l|}{ Reaction } \\
\hline Control group & $59(0,104)$ & $51(0,91)$ & $-14 \%$ & -1.23 & 0.21 & 0.210 \\
\hline Intervention group & $54(8,77)$ & $33(2,76)$ & $-39 \%$ & -2.00 & 0.36 & 0.047 \\
\hline
\end{tabular}

a Percent change in score from pre-programme to post-programme. 